\title{
Evolution equations with parameter in the hyperbolic case
}

\author{
by Jan BocheneK and Teresa Winiarska (Kraków)
}

Abstract. The purpose of this paper is to give theorems on continuity and differentiability with respect to $(h, t)$ of the solution of the initial value problem $d u / d t=$ $A(h, t) u+f(h, t), u(0)=u_{0}(h)$ with parameter $h \in \Omega \subset \mathbb{R}^{m}$ in the "hyperbolic" case.

1. Introduction. We consider the initial value problem

$$
\left\{\begin{array}{l}
\frac{d u}{d t}=A(h, t) u+f(h, t), \quad t \in[0, T], h \in \Omega, \\
u(0)=u_{0}(h) .
\end{array}\right.
$$

It is known that under some assumptions on the family of the operators $\{A(h, t)\}$ and on the function $f$, the problem (1) has the unique solution given by

$$
u(h, t)=U(h, t, 0) u_{0}(h)+\int_{0}^{t} U(h, t, s) f(h, s) d s,
$$

where, for each $h \in \Omega, U$ is the fundamental solution (or evolution system) for problem (1) (cf. [3, Ch. 5]).

Analogously to the papers [5] and [6], where the "parabolic" case of problem (1) was studied, we investigate the continuity and differentiability of the mapping

$$
\Omega \times[0, T] \ni(h, t) \rightarrow u(h, t) \in X,
$$

where the mapping $u$ is given by (2).

2. Stable approximations of the family of operators. This section is based on Krein's monograph [2, Ch. II] and it has the auxiliary character. To simplify notations we assume that the family $\{A(h, t)\}$ considered in the introduction is independent of the parameter $h$.

1991 Mathematics Subject Classification: 34K30, 35B30.

Key words and phrases: evolution problem, stable family of operators, stable approximations of the evolution operator, evolution problem with parameter, hyperbolic case. 
Assuming that $X$ is a Banach space we let $B(X)$ be the Banach space of all linear bounded operators and $\mathcal{C}(X)$ be the vector space of all linear closed operators from $X$ into itself. If $A: X \rightarrow X$ is a linear operator then $D(A), N(A), R(A), \bar{A}, P(A)$ denote the domain, kernel, range, closure and resolvent set of $A$, respectively.

In this section we consider a family of operators $\{A(t)\}, t \in[0, T]$, where $A(t) \in \mathcal{C}(X), D(A(t))=D, \bar{D}=X$ and $0 \in P(A(t))$ for every $t \in[0, T]$.

We investigate the Cauchy problem

$$
\frac{d u}{d t}=A(t) u, \quad u(s)=x, \quad 0 \leq s \leq t \leq T,
$$

where $x \in D$.

Definition 1 ([2, p. 193]). The Cauchy problem (4) is said to be uniformly correct if:

(i) for each $s \in[0, T]$ and any $x \in D$ there exists a unique solution $u=u(t, s)$ of (4) on the interval $[s, T]$,

(ii) the function $u=u(t, s)$ and its derivative $u_{t}^{\prime}$ are continuous in the triangle $\Delta_{T}:=\{(t, s): 0 \leq s \leq t \leq T\}$,

(iii) the solution depends continuously on the initial data.

If the Cauchy problem is uniformly correct, then it is possible to introduce a linear operator $U(t, s)$ for $(t, s) \in \Delta_{T}$ by the formula

$$
U(t, s) x:=u(t, s), \quad(t, s) \in \Delta_{T}, x \in D,
$$

where $u(s, s)=x$. The formula (5) defines the operator $U(t, s)$ on the set $D$ dense in $X$. Since for fixed $(t, s) \in \Delta_{T}$ it is a bounded operator, it admits a continuous extension to the entire space $X$.

It is known (cf. [2, pp. 193-194]) that if for each $x \in D$ the mapping $[0, T] \ni t \rightarrow A(t) x$ is continuous (i.e. the mapping $t \rightarrow A(t)$ is strongly continuous on $D$ ) and the Cauchy problem (4) is uniformly correct, then the fundamental solution $U$ has the following properties:

(a) the mapping $\Delta_{T} \ni(t, s) \rightarrow U(t, s) \in B(X)$ is strongly continuous and $\|U(t, s)\| \leq M$ for $(t, s) \in \Delta_{T}$,

(b) $U(t, t)=I$ and $U(t, s)=U(t, r) U(r, s)$ for $0 \leq s \leq r \leq t \leq T$,

(c) $\frac{\partial}{\partial t} U(t, s) x=A(t) U(t, s) x, \frac{\partial}{\partial s} U(t, s) x=-U(t, s) A(s) x$ for $(t, s) \in$ $\Delta_{T}, x \in D$,

(d) the mappings $\Delta_{T} \ni(t, s) \rightarrow \frac{\partial}{\partial t} U(t, s)$ and $\Delta_{T} \ni(t, s) \rightarrow \frac{\partial}{\partial s} U(t, s)$ are strongly continuous on $D$.

DeFinition $2\left(\left[4\right.\right.$, p. 89]). An operator-valued function $U: \Delta_{T} \ni$ $(t, s) \rightarrow U(t, s) \in B(X)$ satisfying the above conditions (a)-(d) is called the fundamental solution of problem (4). 
It is known (see [2, Ch. II, §2]) that if the operator $A(t)$ is bounded for each $t \in[0, T]$ and the mapping $[0, T] \ni t \rightarrow A(t)$ is strongly continuous, then problem (4) is uniformly correct and so the fundamental solution $U$ for this problem exists.

Definition 3 ([2, p. 199]). If there exists a sequence of bounded and strongly continuous operators $A_{n}(t), t \in[0, T]$, for which

$$
\lim _{n \rightarrow \infty} \sup _{0 \leq t \leq T}\left\|\left[A(t)-A_{n}(t)\right] A(t)^{-1} x\right\|=0, \quad x \in X,
$$

and the fundamental solutions of the problems

$$
\frac{d u}{d t}=A_{n}(t) u, \quad u(s)=x
$$

are uniformly bounded, i.e.,

$$
\left\|U_{n}(t, s)\right\| \leq M
$$

where $M$ does not depend on $n \in \mathbb{N}$ and $(t, s) \in \Delta_{T}$, then we say that the family $\{A(t)\}, t \in[0, T]$, is stably approximated by the sequence $\left\{A_{n}(t)\right\}$.

In $[2, \mathrm{Ch} . \mathrm{II}]$ the following sufficient conditions are given for the family $\{A(t)\}, t \in[0, T]$, to be stably approximated:

(8) the mapping $[0, T] \ni t \rightarrow A(t)$ is strongly continuous in $D$,

$$
\|R(\lambda ; A(t))\|:=\left\|(A(t)-\lambda I)^{-1}\right\| \leq \frac{1}{\lambda+1} \quad \text { for } \lambda \geq 0 .
$$

The sequence $\left\{A_{n}(t)\right\}$ approximating the family $\{A(t)\}, t \in[0, T]$, has the form

$$
A_{n}(t):=-n A(t) R(n ; A(t))
$$

(cf. [2, p. 204]).

Our nearest purpose is to give other sufficient conditions for the family $\{A(t)\}, t \in[0, T]$, to be stably approximated (see Theorems 1 and 2).

Definition 4 ([3, p. 130]). A family $\{A(t)\}, t \in[0, T]$, is called stable if there are constants $M \geq 1$ and $\omega$ (called the stability constants) such that

$$
(\omega, \infty) \subset P(A(t)) \quad \text { for } t \in[0, T]
$$

and

$$
\left\|\prod_{j=1}^{k} R\left(\lambda ; A\left(t_{j}\right)\right)\right\| \leq M(\lambda-\omega)^{-k} \quad \text { for } \lambda>\omega
$$

and for every finite sequence $0 \leq t_{1} \leq \ldots \leq t_{k} \leq T, k \in \mathbb{N}$.

Lemma 1. Let $\{A(t)\}, t \in[0, T]$, be a stable family in the sense of Definition 4. Then the sequence $\left\{A_{n}(t)\right\}$, where $A_{n}(t)$ is defined by (10), is 
uniformly stable, i.e., the stability constants for the operators $A_{n}(t)$ do not depend on $n \in \mathbb{N}$.

Pr o of. From the identity

$$
R\left(\lambda ; A_{n}(t)\right)=\frac{n^{2}}{(n+\lambda)^{2}} R\left(\frac{n \lambda}{n+\lambda} ; A(t)\right)-\frac{1}{n+\lambda} I
$$

we have

$$
\begin{aligned}
\| & \prod_{j=1}^{k} R\left(\lambda ; A_{n}\left(t_{j}\right)\right) \| \\
\leq & \left\|\prod_{j=1}^{k}\left[\frac{n^{2}}{(n+\lambda)^{2}} R\left(\frac{n \lambda}{n+\lambda} ; A\left(t_{j}\right)\right)-\frac{1}{n+\lambda} I\right]\right\| \\
\leq & \mid\left[\frac{n^{2}}{(n+\lambda)^{2}}\right]^{k} M\left(\frac{\lambda n}{n+\lambda}-\omega\right)^{-k} \\
& +\left(\begin{array}{l}
k \\
1
\end{array}\right)\left(\frac{n^{2}}{(n+\lambda)^{2}}\right)^{k-1} \frac{1}{n+\lambda} M\left(\frac{\lambda n}{n+\lambda}-\omega\right)^{-k+1} \\
& +\left(\begin{array}{l}
k \\
2
\end{array}\right)\left(\frac{n^{2}}{(n+\lambda)^{2}}\right)^{k-2} \frac{1}{(n+\lambda)^{2}} M\left(\frac{\lambda n}{n+\lambda}-\omega\right)^{-k+2}+\ldots+\frac{1}{(n+\lambda)^{k}} \mid \\
\leq & M\left(\frac{n}{n+\lambda}\right)^{k}\left(\lambda-\frac{n+\lambda}{n} \omega\right)^{-k} \\
& \times\left[1+\left(\frac{n^{2}}{(n+\lambda)^{2}}\right)^{-1} \frac{1}{n+\lambda}\left(\frac{\lambda n}{n+\lambda}-\omega\right)\right]^{k} \\
= & M\left(\lambda-\frac{n+\lambda}{n} \omega\right)^{-k}\left(1-\frac{\omega}{n}\right)^{k}=M\left(\lambda-\frac{n}{n-\omega} \omega\right)^{-k} .
\end{aligned}
$$

It follows that for $n \geq 2 \omega$, the family $\left\{A_{n}(t)\right\}, t \in[0, T]$, is stable with stability constants $M$ and $2 \omega$ ( $n \geq 2 \omega$ is fixed).

Lemma 2. Let $\{A(t)\}, t \in[0, T]$, be a stable family with stability constants $M$ and $\omega$. If the mapping $[0, T] \ni t \rightarrow A(t) \in B(X)$ is strongly continuous, then the fundamental solution $U$ corresponding to $A(t)$ is strongly continuous in the triangle $\Delta_{T}$ and

$$
\|U(t, s)\| \leq M e^{\omega T} \quad \text { for }(t, s) \in \Delta_{T},
$$

where $M$ and $\omega$ are the stability constants.

Proof. Existence and strong continuity of $U$ follow from boundedness and strong continuity of the mapping $[0, T] \ni t \rightarrow A(t)$. 
In order to prove (13), we start by approximating the family $\{A(t)\}$, $t \in[0, T]$, by piecewise constant families $\left\{A_{\nu}(t)\right\}, t \in[0, T]$, defined as follows. Let $t_{k}^{\nu}:=(k / \nu) T, k=0,1, \ldots, \nu, \nu \in \mathbb{N}$, and let (cf. [3, p. 135])

$$
A_{\nu}(t):= \begin{cases}A\left(t_{k}^{\nu}\right) & \text { for } t_{k}^{\nu} \leq t<t_{k+1}^{\nu}, k=0,1, \ldots, \nu-1, \\ A(T) & \text { for } t=T .\end{cases}
$$

From the strong continuity of $t \rightarrow A(t)$ it follows that

$$
\left\|\left[A(t)-A_{\nu}(t)\right] x\right\| \rightarrow 0 \quad \text { as } \nu \rightarrow \infty
$$

uniformly with respect to $t \in[0, T]$ for each $x \in X$.

Denote by $S_{t}(s), s \geq 0$, the $C_{0}$-semigroup generated by $A(t)$ for $t \in[0, T]$ and let

$$
U_{\nu}(t, s):=\left\{\begin{aligned}
& S_{t_{j}^{\nu}}(t-s) \text { for } t_{j}^{\nu} \leq s \leq t \leq t_{j+1}^{\nu} \\
& S_{t_{k}^{\nu}}\left(t-t_{k}^{\nu}\right)\left[\prod_{j=l+1}^{k-1} S_{t_{j}^{\nu}}(T / \nu)\right] S_{t_{l}^{\nu}}\left(t_{l+1}^{\nu}-s\right) \\
& \quad \text { for } k>l, t_{k}^{\nu} \leq t \leq t_{k+1}^{\nu}, t_{l}^{\nu} \leq s \leq t_{l+1}^{\nu} .
\end{aligned}\right.
$$

From (16) and Theorem 3.1 of [3, p. 135] it follows that $U_{\nu}(t, s)$ is the fundamental solution corresponding to $A_{\nu}(t)$, the mapping

$$
\Delta_{T} \ni(t, s) \rightarrow U_{\nu}(t, s)
$$

is strongly continuous and

$$
\left\|U_{\nu}(t, s)\right\| \leq M e^{\omega(t-s)} \quad \text { for }(t, s) \in \Delta_{T},
$$

where $M$ and $\omega$ are the constants from (12).

From the equality

$$
\frac{\partial}{\partial t} U(t, s) x=A(t) U(t, s) x, \quad x \in X
$$

we obtain

$$
\frac{\partial}{\partial t} U(t, s) x=A_{\nu}(t) U(t, s) x+\left[A(t)-A_{\nu}(t)\right] U(t, s) x .
$$

Hence

$$
U(t, s) x=U_{\nu}(t, s) x+\int_{s}^{t} U_{\nu}(t, \tau)\left[A(\tau)-A_{\nu}(\tau)\right] U(\tau, s) x d \tau
$$

(cf. [2, p. 195, Th. 3.1]) and so we have

$$
\left\|\left[U(t, s)-U_{\nu}(t, s)\right] x\right\| \leq M e^{\omega T} \int_{0}^{T}\left\|\left[A(\tau)-A_{\nu}(\tau)\right] U(\tau, s) x\right\| d \tau .
$$

From (15) and from Lemma 3.7 of $[1$, p. 151] it follows that $\|[U(t, s)-$ $\left.U_{\nu}(t, s)\right] x \| \rightarrow 0$ as $\nu \rightarrow \infty$ uniformly in $(t, s) \in \Delta_{T}$. By (18), this implies (13), i.e. the conclusion of Lemma 2. 
Theorem 1. Suppose that

(i) $\{A(t)\}, t \in[0, T]$, is a stable family in the sense of Definition 4,

(ii) $D(A(t))=D$ does not depend on $t \in[0, T]$,

(iii) the mapping $[0, T] \ni t \rightarrow A(t)$ is strongly continuous,

(iv) $0 \in P(A(t))$ for $t \in[0, T]$.

Then the family $\{A(t)\}, t \in[0, T]$, is stably approximated (cf. Def. 3).

Proof. Define $A_{n}(t)$ by (10) for $n \in \mathbb{N}$. For each fixed $n \in \mathbb{N}$ and $t \in[0, T]$ the operator $A_{n}(t)$ commutes with $A(t)$ on $D$ and $A_{n}(t)$ is a bounded operator on $X$.

Let $x \in D$ be fixed. We have

$$
\begin{aligned}
\|[A(t) & \left.-A_{n}(t)\right] A(t)^{-1} x \| \\
& =\left\|\left[A(t)+n A(t)(A(t)-n)^{-1}\right] A(t)^{-1} x\right\| \\
& =\left\|x+n(A(t)-n)^{-1} x\right\|=\left\|(A(t)-n)^{-1} A(t) x\right\| \\
& \leq\left\|(A(t)-n)^{-1}\right\| \cdot\|A(t) x\| \\
& \leq \frac{M}{n-\omega}\|A(t) x\| \leq \frac{M}{n-\omega} K, \quad \text { where } K=\sup \{\|A(t) x\|: t \in[0, T]\} .
\end{aligned}
$$

This shows that

$$
\left\|\left[A(t)-A_{n}(t)\right] A(t)^{-1} x\right\| \leq M_{1},
$$

where $M_{1}$ does not depend on $n>\omega$ or $t \in[0, T]$. From this estimate we get

$$
\lim _{n \rightarrow \infty} \sup _{0 \leq t \leq T}\left\|\left[A(t)-A_{n}(t)\right] A(t)^{-1} x\right\|=0
$$

for each $x \in D$, where $\bar{D}=X$. By (19) and (20) in view of the BanachSteinhaus theorem (cf. [2, p. 9]), the condition (6) of Definition 3 is satisfied.

From Lemma 2 it follows that the sequence $\left\{A_{n}(t)\right\}$ is uniformly stable with stability constants $M$ and $2 \omega$ for $n \geq 2 \omega$. Using Lemma 2 for each fixed $n \geq 2 \omega$, we obtain

$$
\left\|U_{n}(t, s)\right\| \leq M e^{2 \omega(t-s)} \leq M e^{2 \omega T} .
$$

Theorem 1 is proved.

Lemma 3. Suppose that

(i) the mapping $[0, T] \ni t \rightarrow A(t) x \in X$ is of class $C^{1}$ for $x \in D$,

(ii) $A(t)^{-1} \in B(X)$ exists for $t \in[0, T]$,

(iii) the family $\{A(t)\}, t \in[0, T]$, is stably approximated by the sequence $\left\{A_{n}(t)\right\}$, where $A_{n}(t)$ is defined by $(10)$. 
Then there exists a constant $K$ independent of $n \in \mathbb{N}$ and $(t, s) \in \Delta_{T}$ such that

$$
\left\|A(t) U_{n}(t, s) A(s)^{-1}\right\| \leq K,
$$

where $U_{n}(t, s)$ is the fundamental solution corresponding to $A_{n}(t)$.

Proof. According to Definition 3,

$$
\left\|U_{n}(t, s)\right\| \leq M,
$$

where $M$ does not depend on $n \in \mathbb{N}$ and $(t, s) \in \Delta_{T}$.

Consider the equation (cf. [2, p. 200])

$$
\frac{d y}{d t}=A_{n}(t) y+A^{\prime}(t) A(t)^{-1} y .
$$

By (i) and (ii), the mapping $[0, T] \ni t \rightarrow A^{\prime}(t) A(t)^{-1} \in B(X)$ is strongly continuous. In view of the Banach-Steinhaus theorem we get

$$
\left\|A^{\prime}(t) A(t)^{-1}\right\| \leq C
$$

where $C$ does not depend on $t \in[0, T]$.

Let $V_{n}(t, s)$ be the fundamental solution of $(24)$. We have

$$
V_{n}(t, s)=A(t) U_{n}(t, s) A(s)^{-1}, \quad(t, s) \in \Delta_{T}
$$

(cf. [2, p. 201]). From (23), (25) and (26) it follows that

$$
\left\|V_{n}(t, s)\right\|=\left\|A(t) U_{n}(t, s) A(s)^{-1}\right\| \leq M e^{C M T}=K
$$

(see [2, p. 191]).

Theorem 2. Suppose that

(i) $\{A(t)\}, t \in[0, T]$, is a stable family in the sense of Definition 4,

(ii) $D(A(t))=D$ does not depend on $t \in[0, T]$,

(iii) the mapping $[0, T] \ni t \rightarrow A(t) x \in X$ is of class $C^{1}$ for $x \in D$,

(iv) $A(t)^{-1} \in B(X)$ exists for $t \in[0, T]$.

Then the family $\{A(t)\}, t \in[0, T]$, is stably approximated by the sequence $\left\{A_{n}(t)\right\}$ defined by (10), and the sequence $\left\{U_{n}(t, s)\right\}$ of the fundamental solutions corresponding to $\left\{A_{n}(t)\right\}$ is strongly and uniformly convergent to $U(t, s)$ in $\Delta_{T}$.

Proof. Upon using Theorem 1 and Lemmas 2-4, the proof is analogous to the proof of Theorem 3.11 of $[2$, p. 208]. We omit the details and refer the reader to $[2, \mathrm{Ch}$. II].

From Theorem 2 and [2, Th. 3.6, p. 200] it follows that if the family $\{A(t)\}, t \in[0, T]$, satisfies the assumptions of Theorem 2, then the Cauchy problem

$$
\frac{d u}{d t}=A(t) u, \quad u(s)=x, \quad x \in D, 0 \leq s \leq t \leq T,
$$


has the unique solution given by

$$
u(t)=U(t, s) x,
$$

where $U(t, s)$ is the fundamental solution for (28) defined in Theorem 2.

Remark 1. The set of assumptions (i)-(iii) of Theorem 2 is usually referred to as the "hyperbolic" case in contrast to the "parabolic" case where each $A(t), t \geq 0$, is assumed to be the infinitesimal generator of an analytic semigroup. This terminology is justified by applications of the abstract results to partial differential equations (cf. [3, p. 134]).

3. Dependence of the fundamental solution on parameters. Let $\Omega$ be a compact subset of $\mathbb{R}^{m}$. We shall consider the following initial value problem with a parameter $h \in \Omega$ :

$$
\left\{\begin{array}{l}
\frac{d u}{d t}=A(h, t) u, \quad t \in[0, T], h \in \Omega, \\
u(s)=x, \quad 0 \leq s \leq t \leq T,
\end{array}\right.
$$

where $A: \Omega \times[0, T] \ni(h, t) \rightarrow A(h, t) \in \mathcal{C}(X), D(A(h, t))=D, \bar{D}=X$, $0 \in P(A(h, t))$ for $(h, t) \in \Omega \times[0, T]$ and $x \in D$.

Theorem 3. If , for any $(h, t) \in \Omega \times[0, T], A(h, t)$ is bounded and, for each $x \in X$, the mapping

$$
\Omega \times[0, T] \ni(h, t) \rightarrow A(h, t) x \in X \text { is continuous },
$$

then the mapping

$$
\Omega \times \Delta_{T} \ni(h, t, s) \rightarrow U(h, t, s) x \in X \text { is continuous. }
$$

Proof. It follows from [2, p. 189] that the mapping $\Delta_{T} \ni(t, s) \rightarrow$ $U(h, t, s) x \in X$ is continuous for any fixed $h \in \Omega$ and $x \in X$. Hence, by the Banach-Steinhaus theorem there exists $M_{1}=M_{1}(h) \geq 0$ such that

$$
\|U(h, t, s)\| \leq M_{1} \quad \text { for }(t, s) \in \Delta_{T} .
$$

To prove the theorem it is enough to show that

$$
U(h, t, s) x \rightarrow U\left(h_{0}, t, s\right) x \quad \text { as } h \rightarrow h_{0},
$$

uniformly in $(t, s) \in \Delta_{T}$, for any $x \in X$. Since

$$
\frac{\partial}{\partial t} U(h, t, s) x=A(h, t) U(h, t, s) x \quad \text { for } h \in \Omega,(t, s) \in \Delta_{T}, x \in X,
$$

and $U(h, t, t) x=x$ for $h \in \Omega, t \in[0, T], x \in X$, we have 


$$
\begin{aligned}
\left\|\left[U(h, t, s)-U\left(h_{0}, t, s\right)\right] x\right\| \\
\leq \int_{s}^{t}\left\|\left[A(h, \tau) U(h, \tau, s)-A\left(h_{0}, \tau\right) U\left(h_{0}, \tau, s\right)\right] x\right\| d \tau \\
\leq \int_{s}^{t}\|A(h, \tau)\| \cdot\left\|\left[U(h, \tau, s)-U\left(h_{0}, \tau, s\right)\right] x\right\| d \tau \\
\quad+\int_{s}^{t}\left\|\left[A(h, \tau)-A\left(h_{0}, \tau\right)\right] U\left(h_{0}, \tau, s\right) x\right\| d \tau .
\end{aligned}
$$

By (31) and the Banach-Steinhaus theorem there exists $M>0$ such that $\|A(h, t)\| \leq M$. Thus,

$$
\begin{aligned}
\left\|\left[U(h, t, s)-U\left(h_{0}, t, s\right)\right] x\right\| \leq & M \int_{0}^{T}\left\|\left[U(h, \tau, s)-U\left(h_{0}, \tau, s\right)\right] x\right\| d \tau \\
& +\int_{0}^{T}\left\|\left[A(h, \tau)-A\left(h_{0}, \tau\right)\right] U\left(h_{0}, \tau, s\right) x\right\| d \tau .
\end{aligned}
$$

By Gronwall's inequality

$$
\left\|\left[U(h, t, s)-U\left(h_{0}, t, s\right)\right] x\right\| \leq e^{T M} \int_{0}^{T}\left\|\left[A(h, \tau)-A\left(h_{0}, \tau\right)\right] U\left(h_{0}, \tau, s\right) x\right\| d \tau .
$$

By (31) the operators $A(h, \tau)-A\left(h_{0}, \tau\right)$ converge strongly and uniformly in $\tau \in[0, T]$ to zero as $h \rightarrow h_{0}$, on the entire space $X$. This means that they converge to zero on the compact set of values of the continuous functions $U\left(h_{0}, \tau, s\right) x$. It follows that the functions

$$
\left[A(h, \tau)-A\left(h_{0}, \tau\right)\right] U\left(h_{0}, \tau, s\right) x
$$

converge to zero uniformly in $(\tau, s) \in \Delta_{T}$ (cf. [1, p. 151]). Hence $\lim _{h \rightarrow h_{0}} U(h, t, s) x=U\left(h_{0}, t, s\right) x$ uniformly in $(t, s) \in \Delta_{T}$.

Definition 5. A family $\{A(h, t)\},(h, t) \in \Omega \times[0, T]$, is said to be $u n i$ formly stably approximated with respect to $h \in \Omega$ if there exists a sequence $\left\{A_{n}(h, t)\right\}$ of bounded linear operators $A_{n}(h, t): X \rightarrow X, n=1,2, \ldots$, such that

(i) the mapping $\Omega \times[0, T] \ni(h, t) \rightarrow A_{n}(h, t) x \in X$ is continuous for $x \in X, n=1,2, \ldots$,

(ii) $\lim _{n \rightarrow \infty}\left\{\sup \left\|\left[A_{n}(h, t)-A(h, t)\right] A(h, t)^{-1} x\right\|:(h, t) \in \Omega \times[0, T]\right\}=0$ for $x \in X$ and the sequence $\left\{U_{n}(h, t, s)\right\}$ of fundamental solutions of (30) with $A(h, t)=A_{n}(h, t), n=1,2, \ldots$, is uniformly bounded, i.e. there exists 
$K>0$ such that

$$
\left\|U_{n}(h, t, s)\right\| \leq K \quad \text { for } h \in \Omega,(t, s) \in \Delta_{T}, n=1,2, \ldots
$$

Definition 6. We say that a family $\{A(h, t)\},(h, t) \in \Omega \times[0, T]$, is uniformly stable in $\Omega$ if

(i) $\{A(h, t)\}$ is stable (in the sense of Def. 4) for any $h \in \Omega$,

(ii) the stability constants $M, \omega$ are independent of $h$.

TheOREM 4. Suppose that

(i) the family $\{A(h, t)\},(h, t) \in \Omega \times[0, T]$ is uniformly stably approximated by $\left\{A_{n}(h, t)\right\},(h, t) \in \Omega \times[0, T]$,

(ii) the mapping $\Omega \times[0, T] \ni(h, t) \rightarrow A(h, t) x \in X$ is continuous for $x \in D$,

(iii) the mapping $[0, T] \ni t \rightarrow A(h, t) x \in X$ is of class $C^{1}$ for $h \in \Omega$, $x \in D$,

(iv) $A_{n}(h, t)$ commutes with $A(h, t)$ for $n \in \mathbb{N},(h, t) \in \Omega \times[0, T]$,

(v) $\left\{U_{n}(h, t, s)\right\}$ strongly and uniformly converges to $U(h, t, s)$ in $\Omega \times \Delta_{T}$.

Then $U(h, t, s)$ is the fundamental solution of the problem (30) and the mapping $(h, t, s) \rightarrow U(h, t, s) x$ is continuous.

Proof. It follows from Theorem 3.6 of $[2$, p. 200] that the problem (30) is uniformly correct and, for $h \in \Omega, U(h, t, s)$ is its fundamental solution. By (i), the assumptions of Theorem 3 are satisfied. Thus, for $n \in \mathbb{N}$, the mapping $(h, t, s) \rightarrow U_{n}(h, t, s) x$ is continuous and the assumption (v) ends the proof.

TheOREM 5. Suppose that

(i) $\{A(h, t)\},(h, t) \in \Omega \times[0, T]$, is stable uniformly in $h \in \Omega$,

(ii) the mapping $\Omega \times[0, T] \ni(h, t) \rightarrow A(h, t) x \in X$ is continuous for $x \in D$,

(iii) the mapping $[0, T] \ni t \rightarrow A(h, t) x \in X$ is of class $C^{1}$ for $h \in \Omega$, $x \in D$.

Then the problem (30) has, for any $h \in \Omega$, exactly one solution $u(h, \cdot)$ which is given by $u(h, t)=U(h, t, s) x$, where $U(h, t, s)$ is the fundamental solution of (30). Moreover, the mapping $\Omega \times \Delta_{T} \ni(h, t, s) \rightarrow U(h, t, s) x \in X$ for $x \in X$ is continuous.

Proof. Since for any $h \in \Omega$, the family $\{A(h, t)\}$ satisfies the assumptions of Theorem 2 , it is stably approximated and the approximating sequence is given by

$$
A_{n}(h, t)=-n A(h, t) R(n ; A(h, t))=-n I-n^{2} R(n ; A(h, t)) .
$$


By (i),

$$
\|R(n ; A(h, t))\| \leq \frac{M}{n-\omega}
$$

and so $R(n ; A(h, t))$ is bounded uniformly in $(h, t) \in \Omega \times[0, T]$, for any fixed $n \in \mathbb{N}$. Hence the mapping $(h, t) \rightarrow A_{n}(h, t) x$ for $x \in X$ is continuous (see [2, p. 176]), where $A_{n}(h, t)$ is given by (33). By Theorem 3 the mapping

$$
(h, t, s) \rightarrow U_{n}(h, t, s) x \quad \text { for } x \in X, n=1,2, \ldots,
$$

is continuous, where $U_{n}(h, t, s)$ is the fundamental solution of (30) with $A(h, t)=A_{n}(h, t)$ given by (33). By Theorem 2 the sequence $\left\{U_{n}(h, t, s)\right\}$ is strongly and uniformly convergent to $U(h, t, s)$ in $\Delta_{T}$, for $h \in \Omega$. Since the family $\{A(h, t)\}$ is uniformly stably approximated with respect to $h \in \Omega$, similarly to the proof of Theorem 3.11 in [2] we conclude that $U_{n}(h, t, s) x \rightarrow$ $U(h, t, s) x$ uniformly in $(h, t, s) \in \Omega \times \Delta_{T}$.

4. Dependence on parameter of solutions to problem (1). It is well known that under suitable assumptions the solution of problem (1) is given by

$$
u(h, t)=U(h, t, 0) u_{0}(h)+\int_{0}^{t} U(h, t, s) f(h, s) d s .
$$

TheOREM 6. Suppose that

(i) the family $\{A(h, t)\}$ satisfies the assumptions of Theorem 4,

(ii) the mapping $\Omega \ni h \rightarrow u_{0}(h) \in X$ is continuous,

(iii) the mapping $\Omega \times[0, T] \ni(h, t) \rightarrow f(h, t) \in X$ is continuous.

Then the function $u$ given by (34) is continuous in $\Omega \times[0, T]$.

Pr o o f. By Theorem 4 the mapping $\Omega \times \Delta_{T} \ni(h, t, s) \rightarrow U(h, t, s) x \in X$ for $x \in X$ is continuous and so Theorem 6 is now a simple consequence of Theorem 1 of [5].

Corollary. If the family $\{A(h, t):(h, t) \in \Omega \times[0, T]\}$ satisfies the assumptions of Theorem 5 and the mappings $\Omega \ni h \rightarrow u_{0}(h) \in X$ and $\Omega \times[0, T] \ni(h, t) \rightarrow f(h, t) \in X$ are continuous then the function given by (34) is continuous in $\Omega \times[0, T]$.

Indeed, it is a simple consequence of Theorems 5 and 6 .

TheOREm 7. Let the assumptions of Theorem 4 be satisfied. Suppose that $\Omega \subset \mathbb{R}, h_{0}$ is an interior point of $\Omega$ and

(i) $u(h, \cdot) \in C([0, T] ; X)$ is a solution of the problem (1),

(ii) the mappings $\Omega \ni h \rightarrow A(h, \cdot) x \in C([0, T] ; X), \Omega \ni h \rightarrow f(h, \cdot) \in$ $C([0, T] ; X)$ and $\Omega \ni h \rightarrow u_{0}(h) \in X$ are differentiable at $h_{0}$. 
Then the mapping $\Omega \ni h \rightarrow u(h, \cdot) \in C([0, T] ; X)$ is differentiable at $h_{0}$ and

$$
\begin{aligned}
u^{\prime}\left(h_{0}, t\right)= & U\left(h_{0}, t, 0\right) u_{0}^{\prime}\left(h_{0}\right) \\
& +\int_{0}^{t} U\left(h_{0}, t, s\right)\left[f^{\prime}\left(h_{0}, s\right)-A^{\prime}\left(h_{0}, s\right) u\left(h_{0}, s\right)\right] d s,
\end{aligned}
$$

where " 1 " denotes differentiation with respect to $h$.

Proof. Since $u(h, \cdot)$ is a solution of the problem (1), the function

$$
\omega(h, t)=\frac{u(h, t)-u\left(h_{0}, t\right)}{h-h_{0}} \quad \text { for } h \neq h_{0}
$$

is for $h \neq h_{0}$ a solution of the problem

$$
\left\{\begin{array}{l}
\frac{d v}{d t}=A(h, t) v+F(h, t), \\
v(0)=\omega_{0}(h)
\end{array}\right.
$$

where

$$
\begin{gathered}
F(h, t)= \begin{cases}\frac{f(h, t)-f\left(h_{0}, t\right)}{h-h_{0}}-\frac{A(h, t)-A\left(h_{0}, t\right)}{h-h_{0}} u\left(h_{0}, t\right) & \text { for } h \neq h_{0}, \\
f^{\prime}\left(h_{0}, t\right)-A^{\prime}\left(h_{0}, t\right) u\left(h_{0}, t\right) & \text { for } h=h_{0},\end{cases} \\
\omega_{0}(h)= \begin{cases}\frac{u_{0}(h)-u_{0}\left(h_{0}\right)}{h-h_{0}} & \text { for } h \neq h_{0}, \\
u_{0}^{\prime}\left(h_{0}\right) & \text { for } h=h_{0} .\end{cases}
\end{gathered}
$$

By (ii) the mapping

$$
(h, t) \rightarrow \begin{cases}\frac{f(h, t)-f\left(h_{0}, t\right)}{h-h_{0}} & \text { for } h \neq h_{0}, \\ f^{\prime}\left(h_{0}, t\right) & \text { for } h=h_{0},\end{cases}
$$

is continuous. We have

$$
\begin{aligned}
& \frac{A(h, t)-A\left(h_{0}, t\right)}{h-h_{0}} u\left(h_{0}, t\right) \\
& \quad=\frac{A(h, t)-A\left(h_{0}, t\right)}{h-h_{0}} A\left(h_{0}, 0\right)^{-1} A\left(h_{0}, 0\right) A\left(h_{0}, t\right)^{-1} A\left(h_{0}, t\right) u\left(h_{0}, t\right) .
\end{aligned}
$$

Since

$$
A\left(h_{0}, t\right) u\left(h_{0}, t\right)=\frac{d u\left(h_{0}, t\right)}{d t}-f\left(h_{0}, t\right)
$$

and by Definition 1, the mapping

$$
[0, T] \ni t \rightarrow A\left(h_{0}, t\right) u\left(h_{0}, t\right) u
$$

is continuous. Also, the mapping

$$
[0, T] \ni t \rightarrow A\left(h_{0}, t\right) A\left(h_{0}, t\right)^{-1} u
$$


is continuous (cf. [2, Lemma 1.5]). Therefore

$$
(h, t) \rightarrow \begin{cases}\frac{A(h, t)-A\left(h_{0}, t\right)}{h-h_{0}} u\left(h_{0}, t\right) & \text { for } h \neq h_{0}, \\ A^{\prime}\left(h_{0}, t\right) u\left(h_{0}, t\right) & \text { for } h=h_{0},\end{cases}
$$

is continuous. By Theorem 6 the mapping

$$
\widetilde{\omega}(h, t):=U(h, t, 0) \omega_{0}(h)+\int_{0}^{t} U(h, t, s) F(h, s) d s
$$

is continuous and

$$
\widetilde{\omega}(h, t)= \begin{cases}\omega(h, t) & \text { for } h \neq h_{0} \\ u^{\prime}\left(h_{0}, t\right) & \text { for } h=h_{0} .\end{cases}
$$

Therefore

$$
\begin{aligned}
u^{\prime}\left(h_{0}, t\right)= & U\left(h_{0}, t, 0\right) u_{0}^{\prime}\left(h_{0}\right) \\
& +\int_{0}^{t} U\left(h_{0}, t, s\right)\left[f^{\prime}\left(h_{0}, s\right)-A\left(h_{0}, s\right) u\left(h_{0}, s\right)\right] d s .
\end{aligned}
$$

COROLlaRY 2. If for any $h \in \Omega$ the assumptions of Theorem 7 are satisfied, then the mapping

$$
\Omega \ni h \rightarrow u(h, \cdot) \in C([0, T] ; X)
$$

is differentiable and

$$
u^{\prime}(h, t)=U(h, t, 0) u_{0}^{\prime}(h)+\int_{0}^{t} U(h, t, s) F_{1}(h, s) d s,
$$

where $F_{1}(h, s)=f^{\prime}(h, s)-A^{\prime}(h, s) u(h, s)$.

R e mark 1. Let the assumptions of Theorem 4 be satisfied. If for any $h \in \Omega$ the mapping $[0, T] \ni t \rightarrow f(h, t) \in X$ is of class $C^{1}$, then the function $u$ given by (34) is the unique solution of the problem (1) (see [4, Th. 4.52]).

R e mark 2. Similarly to [6] one can prove theorems on higher regularity of the solution of problem (1).

\section{References}

[1] T. Kato, Perturbation Theory for Linear Operators, Springer, 1980.

[2] S. G. Krein, Linear Differential Equations in Banach Space, Transl. Amer. Math. Soc. 29, Providence, R.I., 1971.

[3] A. Pazy, Semigroups of Linear Operators and Applications to Partial Differential Equations, Springer, 1983.

[4] H. Tanabe, Equations of Evolution, Pitman, 1979. 
[5] T. Winiarska, Parabolic equations with coefficients depending on t and parameters, Ann. Polon. Math. 51 (1990), 325-339.

[6] - Regularity of solutions of parabolic equations with coefficients depending on $t$ and parameters, ibid. 56 (1992), 311-317.

Institute of Mathematics

Technical University of Kraków

Warszawska 24

31-155 Kraków, Poland

E-mail: u-2@institute.pk.edu.pl

Reçu par la Rédaction le 8.12.1994

Révisé le 27.4.1995 